\title{
Dislocations in Polymer Crystals
}

\author{
H. D. Keith ${ }^{1}$ and Elio Passaglia
}

(April 20, 1964)

\begin{abstract}
The role that dislocations are likely to play in chain-folded polymer crystals is examined, particularly with regard to their influence on plastic deformation. It is assumed that primary bonds cannot be broken in backbone molecular chains; and this restriction, together with further restrictions brought about by chain folding, limits substantially the number of permissible dislocations and glide processes. It is shown that deformations of appreciable magnitude in chain-folded polymer crvstals almost certainly cannot be attributed solely to dislocation mechanisms. Crystals of the $n$-alkanes and "extended chain" polymer crystals are also discussed briefly.
\end{abstract}

\section{Introduction}

The recent discoveries that high polymers are capable of forming chain-folded lamellar single crystals when deposited from dilute solution, and that lamellar crystals of a similar nature are present in many bulk polymers [1], ${ }^{2}$ have aroused considerable interest in the growth and properties of chain-folded molecular crvstals. These crvstals have been most widely studied in polyethylene, but attention has been confined, for the most part, to what may be considered properties of the ideal crystal. That is to say, for the interpretation of most observations recorded to date, it is sufficient to represent a lamellar single crystal as a perfectly alined array of uniformly folded molecules which is bounded on two sides by flat surfaces consisting of regularly packed molecular folds. Properties sensitive to defect structures within real crystals have not yet been investigated thoroughly or systematically. It is known that giant screw dislocations are commonly present during growth and often give rise to growth spirals or terraced growth pyramids, and that Moiré patterns occasionally reveal features probably indicative of the presence of edge dislocations. But there is little evidence beyond this relating to possible defects in the crystals, or to frequencies of their occurrence.

However, there is precedence from experience with metallic and ionic solids for the view that such defects may contribute significantly to the physical properties of polymer crystals, and particularly to processes of annealing, recrystallization and plastic deformation. As early as 1958, the possible role of dislocation mechanisms in the deformation of polyethylene was discussed extensively by Frank, Keller, and O'Connor [2]; however, the influence of chain folding was not considered. More recently, dislocation mechanisms have been proposed to account for plastic flow in nylons [3] and for the thickening

1 Bell Telephone Laboratories, Murray Hill, N.J.

2 Italized figures in brackets indicate the literature references at the end of this paper. of polyethylene crystals on annealing [4] but, in both cases, in terms of dislocations of new types which have no direct counterparts in simpler solids. Our purpose here is to re-examine the part that dislocations might play in crystalline polymers, now taking chain folding into account. We shall point out that the applicability of dislocation concepts to these materials can easily be exaggerated if not examined critically. We shall be concerned principally with the enumeration of dislocations which are geometrically permissible in polymer crystals and with assessing the likelihood of their participating in plastic deformation. Indeed, not much more is possible at present since neither the energies associated with dislocations nor the stresses required to move them can be estimated with confidence in these systems.

The prime consideration to be borne in mind is that no dislocation is allowed which demands that primary bonds be broken for its formation or motion. As we shall see, this limits the number of permissible dislocations and slip systems quite substantially. We shall see also that further restrictions are imposed by chain folds at external surfaces of the crystals. For simplicity we proceed in three stages. First we consider an "infinite chain" crystal, that is, a crystal composed of very long chains which are not folded. Secondly, we consider crystals of long chain hydrocarbons ( $n$-alkanes) which consist of layers each having a structure differing from the infinite chain crystal only insofar as the chains. (which are of uniform length) are relatively short. Finally, we consider chain-folded polymer crystals. As a specific model for discussion, we assume the crystals in each instance to have orthorhombic subcells identical to that of polyethylene [5]. As a further simplification, we limit our discussion in the cases of hydrocarbons and polymers to flat, as distinct from pyramidal, crystals $[6,7]$. Although our results are directly applicable only to a small range of materials, they will nevertheless serve to illustrate how dislocation processes are restricted in long chain molecular crystals in general. Extension of our analysis to other structures would in most instances be a simple matter. 


\section{2. "Infinite Chain" Crystals}

Under the restriction that primary bonds must not be broken during deformation, or that chains do not remain bent after deformation, the only possible slip planes are $(h k 0)$ planes. But, of these, we may confine our attention to the more closely packed low index planes (100), (010), and (110), for it is unlikely that planes of higher index will be effective as slip planes. It is now relatively easy to enumerate the dislocations which can give rise to slip on these planes.

In table 1 we have listed possible screw and edge dislocations, but limiting ourselves, for simplicity, to dislocations of unit strength (Burgers vector equal to a primitive translation of the lattice), and omitting other dislocations obtainable from those listed by trivial change, as in the sign of a Miller index or a Burgers vector. Given in the table are the orientations of the Burgers vector, $b$, and of the line, $l$, of each dislocation, together with its associated glide plane. For the four screw dislocations $(1-4), b$ and $l$ are, of course, identical and, in all respects save one, the entries in the table are arrived at by simple considerations such as would apply in a metal crystal $[8,9]$. The notable exception is the assignment of specific glide planes to the screw dislocations 2,3 , and 4 . Whereas a screw dislocation in a metal or an ionic crystal can glide in any plane of the zone defined by the dislocation line as axis, dislocations 2,3 , and 4 in an infinite chain crystal can glide easily only in the planes we have indicated. If they were to glide in any other planes they would leave behind in their wakes planes of disorder in which molecular chains suffer a sigmoid bending (see later); the only alternative to such a bending would be the breaking of bonds. We shall now consider each of the dislocations listed in more detail.

TABLE 1.

\begin{tabular}{|c|c|c|c|}
\hline \multicolumn{4}{|c|}{ Screw dislocations } \\
\hline & Burgers vector & Dislocation line & Glide plane \\
\hline (1) & [001] & [001] & Any plane of the \\
\hline $\begin{array}{l}(2) \\
(3)\end{array}$ & $\begin{array}{l}{[100]} \\
{[010]}\end{array}$ & $\begin{array}{l}{[100]} \\
{[010]}\end{array}$ & $\begin{array}{l}(010) \\
(100)\end{array}$ \\
\hline (4) & [110] & {$[110]$} & (i10) \\
\hline \multicolumn{4}{|c|}{ Edge dislocations } \\
\hline $\begin{array}{l}(5) \\
(6)\end{array}$ & {$\left[\begin{array}{l}{[100]} \\
{[010]}\end{array}\right.$} & {$\left[\begin{array}{l}{[001]} \\
{[001]}\end{array}\right.$} & $\begin{array}{l}(010) \\
(100)\end{array}$ \\
\hline (7) & {$[110]$} & [001] & $(1 \overline{10})$ \\
\hline $\begin{array}{l}(8) \\
(9)\end{array}$ & {$[001]$} & {$[100]$} & $\begin{array}{l}(010) \\
(100)\end{array}$ \\
\hline (10) & [001] & [110] & (110) \\
\hline
\end{tabular}

\subsection{Screw Dislocations}

Screw dislocations of type 1 are unique in the infinite chain crystal in that they are the only screw dislocations possible which do not require any distortion or bending of molecular chains. They could exist, indeed, in a crystal composed of closely packed rigid rods. These dislocations, therefore, are free to glide in any plane of the [001] zone; but, of course, close-packed planes will be favored. Except in that they have a relatively small Burgers vector $(2.54 \AA)$ they are topologically similar to the giant screw dislocations which contribute significantly to crystal growth in polyethylene lamellae formed in dilute solution [1].

As already mentioned, dislocations of types 2, 3, and 4 are different. Here the dislocation lines are perpendicular to the chains and, since the stress field of a screw dislocation is confined to shears acting along the direction of the dislocation line $[8,9]$, there must be associated with these dislocations shear stresses which cause the chains to bend. For example, let us now consider a dislocation of type 2 , shown schematically in figure 1 . This dislocation can glide in the (010) plane without doing more than causing the shear field (whose form is obvious from fig. 1) to move with it, and at the same time causing chains to slip past one another over the plane swept out by the glide motion. But, if it were to glide in the (001) plane, say (that is, in or out of the plane of the paper in fig. 1), it would leave behind it in the region swept out by glide a plane of sigmoid bends (shaded area in fig. 2). In other words, such a motion of the dislocation would not only cause chains to slip past one another in (010) planes, but would also allow the upper part of the crystal to move relative to the lower part (by a translation $b$ ) only at the expense of bending every chain in that part of the crystal through which the dislocation has passed. It is clear on energetic grounds that such a slip process will not figure prominently in the behavior of real crystals. Moreover, such a process implies that a stress exists over the whole plane of bent bonds instead of being localized near the dislocation line. It is a plane defect rather than a line defect and hence difficult to classify as a perfect dislocation $[8,9]$.

A word may be said in passing about the means whereby chains close to a dislocation line may bend in the cases of dislocations of types 2,3 , and 4 (see again fig. 1). In our opinion, this distortion is most likely brought about by bond stretching and by slight distortion of valence bond angles. We are led to this view by noting that, whereas rotations about bonds would be favored in terms of intra-chain energy, they would not lead to a slight elongation of the chain as is required (quite the opposite) and, in addition, they would give rise to larger inter-chain conflicts.

\subsection{Edge Dislocations}

The six edge dislocations listed in table 1 represent the only ones likely to participate in slip processes in an infinite chain crystal. The first three (5-7) involve no bond distortion and, like screw dislocations of type 1, could exist in a lattice of rigid rods. They require little comment, other than to remark (a) that their energies of formation (proportional to $b^{2}$ ) increase in the order 6,5 , and 7 ; (b) that a dislocation 


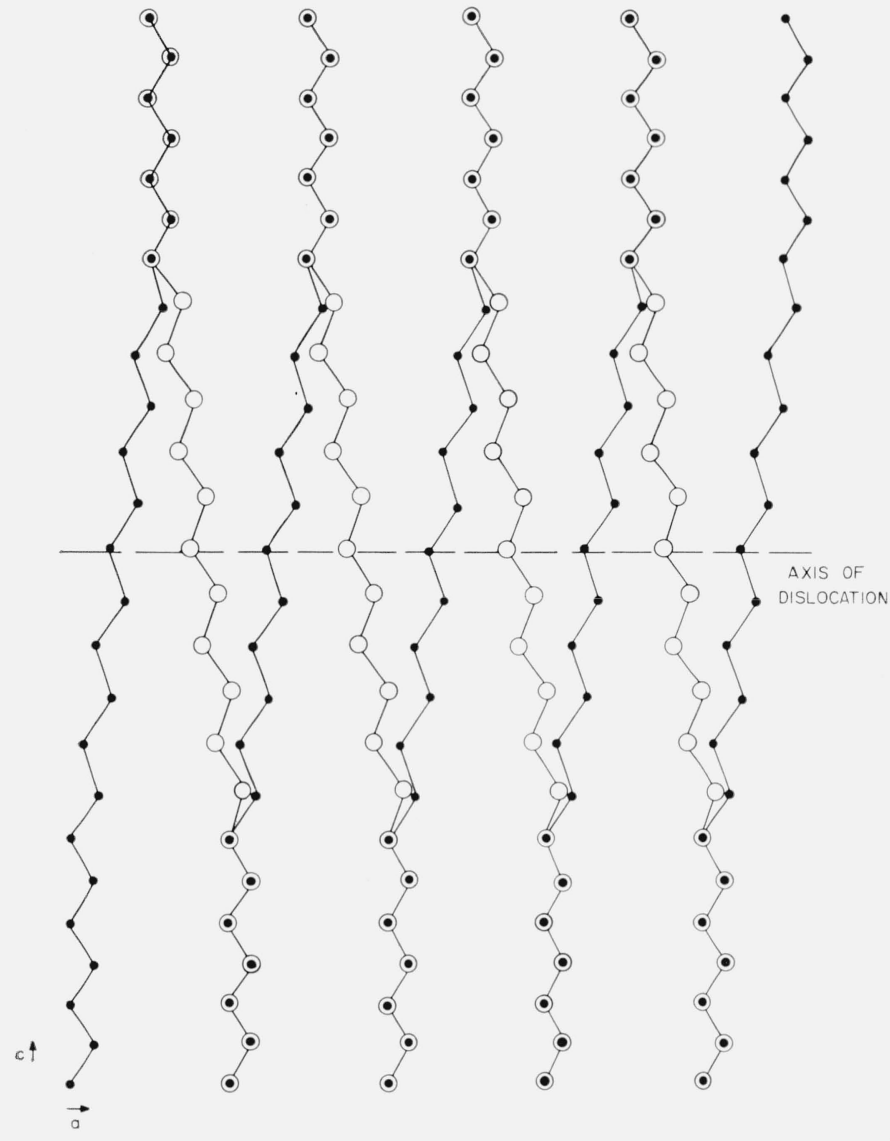

FIgURE 1. A schematic representation of a screw dislocation with [100] Burgers vector in polyethylene.

The chains are projected on the (010) plane and for clarity only the chains at the corners of the unit cell are shown.

of type 7 might well dissociate into a pair of partial dislocations [2]; and (c) that, regarding an edge dislocation of unit strength as arising by the insertion of a half-plane of molecules into an otherwise perfect lattice, then, on account of the "body centered" character of the unit cell we are considering, what must be inserted is two half-planes which may be of (110), (100), or (010) type. With reference to (b) it may be pointed out that dissociation of a dislocation of type 7 into partials is limited by the energy required to generate a ribbon of stacking fault between the separated partials. Although this energy might be expected to be appreciable for polyethylene, the possibility exists that it may become quite small at elevated temperatures because of rotation (perhaps even fairly free rotation) of molecular chains in the lattice. Dislocations might then split readily into partials at higher temperatures and, despite unfavorable energetics, these partials may remain separated, for the most part, on cooling again to room temperature. ${ }^{3}$

The remaining dislocations (8-10) require more detailed discussion. These dislocations involve

3 Note added in proof: Recent studies by V. F. Holland (J. Appl. Phys. 35, (1964)) show that appreciable densities of such partials are produced in chain-folded polyethylene crystals by heat treatment.

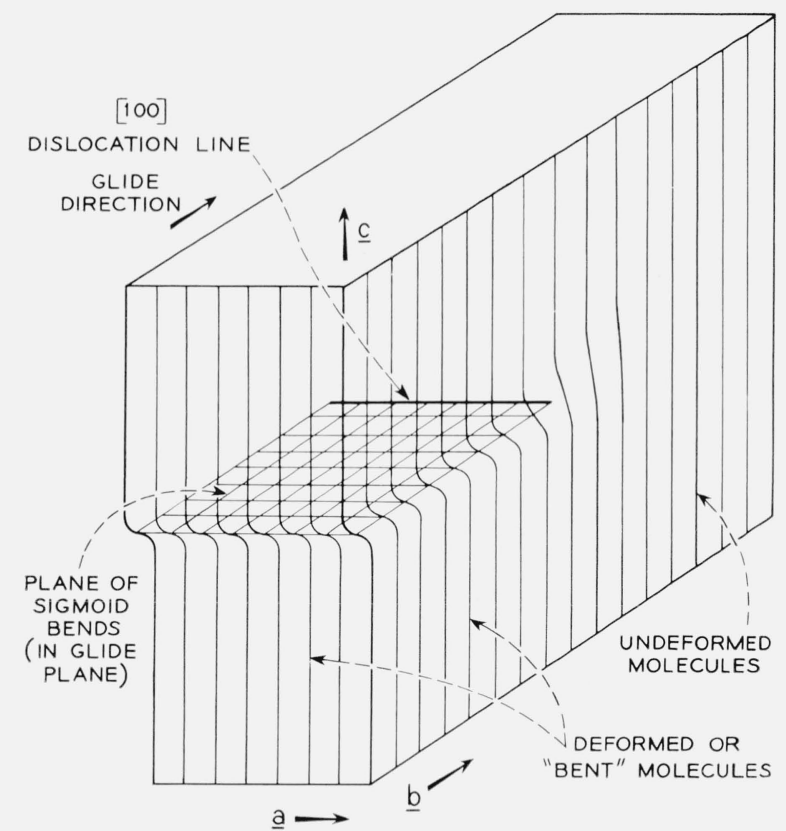

Figure 2. A schematic representation of the effect of a screw dislocation with [100] Burgers vector gliding in a (001) plane.

Notice that a plane of sigmoid bends is left as a result of this motion, which accordingly is considered to be energetically most unlikely.

strains in the chain direction which are opposed by relatively strong forces, and would inevitably be ones with which substantial line energies must be associated. Indeed, we are uncertain of the likelihood of their ever being formed. But, in the absence of these dislocations, it may be noted, the only dislocation mechanism which would lead to slip in the [001] direction under the action of a simple shear on an $(h k 0)$ plane would involve the glide of screw dislocations of type 1 .

We suggest that, if $b=[001]$ edge dislocations do occur, they are probably formed by bond stretching and bond angle distortion. Dislocations as recently proposed to account for slip bands in nylon $66[3]$ we consider to be much less likely. In the latter, planes of molecules are considered to "bulge" at the dislocations, thus giving rise to large cavities in the crystal. In negotiating these bulges, molecules must undergo rotations about bonds such as would give rise to severe inter-chain conflicts (this is easily demonstrated with a Hirschfelder model). The longer the repeat distance along the chain ( $\sim 17 \mathrm{~A}$ for nylon 66 ) the larger the cavities and the more severe the conflicts become; at the same time, the resistance to glide motion increases correspondingly. In these terms it would appear that edge dislocations whose Burgers vectors are parallel to a molecular chain axis are likely only in polymers in which the chemical repeat distance is fairly short.

\section{Long Chain Hydrocarbon Crystals}

In this section we again use as a model for discussion hydrocarbon crystals of orthorhombic structure. For these crystals two unit cells may be 
distinguished; a primary unit cell which incorporates in its $c$ direction the full length of two hydrocarbon chains and a subcell which in its $c$ direction incorporates two $\mathrm{CH}_{2}$ groups and has a length 2.54 $\AA[10]$. The $a$ and $b$ dimensions are the same for both cells and apart from slight variations in $a$ and $b$, the subcell is identical with those of the infinite chain crystal and of polyethylene.

The uniform length of the hydrocarbon chains clearly introduces as a new glide plane the plane on either side of which lie terminal methyl groups of the extended molecules. In the system defined by the primary cell these are (002) planes [10]. The existence of this glide plane, across which there are no molecular ties, obviously brings several new dislocations into play.

Screw dislocations similar to type 1 , but with Burgers vectors equal to integral multiples of that of dislocation 1, can occur in hydrocarbon crystals just as they might arise in an infinite chain crystal. But now, as a special case, these would include the screw dislocation which gives rise to the well known growth steps in hydrocarbon crystals and has a Burgers vector equal to the length of one or more extended molecules. In addition to this dislocation, there are dislocations which may glide in the new glide plane. These are the screw dislocation 2-4 listed earlier, and three new edge dislocations with Burgers vectors [100], [010], [110], and lines [010], [100], and [130], respectively. For the third of these to be strictly an edge dislocation, its line must be normal to $b$; it must therefore lie close to the $[1 \overline{3} 0]$ direction in this structure. Any of these dislocations cause individual layers of molecules to slip over one another, leading to a "card-deck" type of deformation which, a priori, would seem a likely process in these crystals.

\section{Chain-Folded Polyethylene Single Crystals}

Proceeding now to the case of a polyethylene single crystal, we recognize immediately that slip processes must be limited in many cases by the fact that neighboring lengths of chain may be connected by virtue of molecular folding. Moreover, the geometry of this folding will be an important consideration. To illustrate this, we consider a crystal with molecules folded regularly in $\{110\}$ planes, and in figure 3 we show a boundary between different fold domains for one of the possible folding regimes of this type [11].

It is evident in this case that the only planes on which repeated slip is possible without disruption of the structure are $\{110\}$ planes, and of these there is but one suitable plane in each domain. This is the fold plane [11], which lies parallel to the growth front by the advance of which the particular domain in question was initially formed. Since there are no primary bond connections between molecular segments in a given fold plane and segments in either of the adjacent fold planes, the given plane is clearly

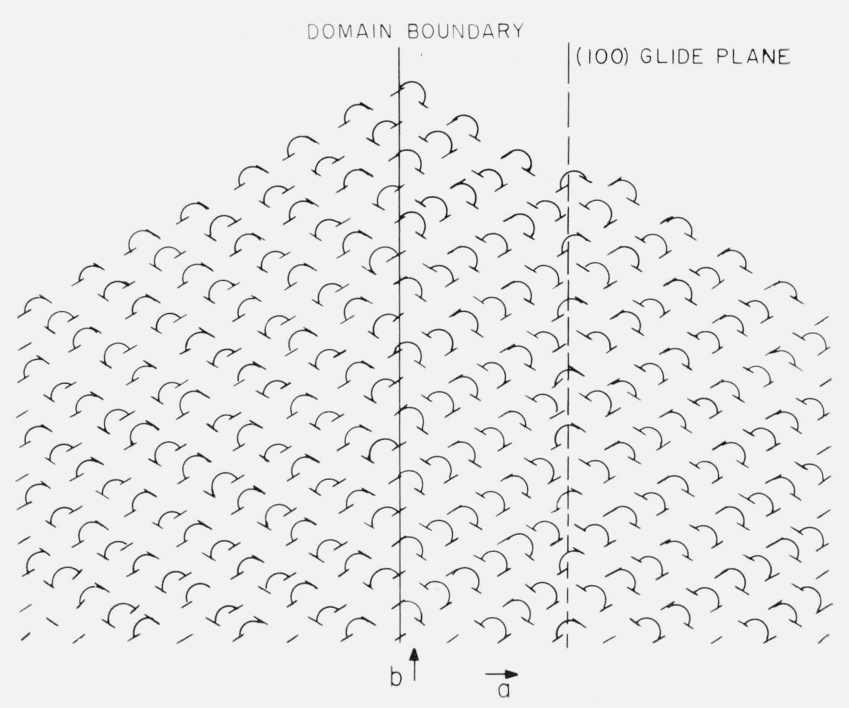

Figure 3. A schematic representation of the chain-folded surface of a lamellar polyethylene crystal.

The only true glide planes are the fold planes. In the right hand half of the crystal a dislocation with [010] Burgers vector has glided in a (100) plane and resulted in a modification of the folding scheme. Note that subsequent slip in this plane is impossible without a drastic rearrangement of the molecules and of the fold geometry.

a possible glide plane. The dislocations which may glide in this plane are obviously the same as those which can glide in the corresponding plane of an infinite chain crystal and have already been listed in table 1. However, any departure from regular folding which gives rise to connectivity between fold planes is potentially a barrier to glide. Fold domain boundaries, in particular, are impenetrable barriers to the passage of dislocations in this structure other than screw dislocations of type 1 which could conceivably approach a boundary along a fold plane in one domain, change direction at the boundary and then glide in the (different) fold plane of the adjacent domain. For example, a screw dislocation might approach the boundary in figure 3 from the left along a (1) 0 ) plane and, having passed through the boundary, continue to the right along a (110) plane. Favorable stresses for generating such a motion might well arise in the drying down of pyramidal crystals (see later), but it is difficult to see how they would otherwise be produced in any concerted fashion.

As is evident from figure 3 , a limited amount of slip is possible in [010] or conceivably in [100] directions. However, these motions are restricted to translations of one unit cell and, even then, to translations in specific directions. For, if (110) is the fold plane, as in the right of figure 3, then the part of the crystal lying to the right of a (010) slip plane may move upward by one unit with respect to the remainder of the crystal because the folds may be rearranged to lie in the [110] rather than the $[1 \overline{1} 0]$ direction. But these folds cannot easily be stretched the distance required either for slip in the opposite direction, or for multiple slip processes, to occur. 
An interesting situation arises if slip takes place in the $c$ direction in a plane such as the (010) plane and, in principle, this could be caused by the glide of dislocations of types 1 or 9 . Because of the continuity of folded molecules, configurations such as shown in figure 4 must result. Motion of these dislocations in adjacent planes, successively, might occur in the collapse of pyramidal or ridged crystals which become flat as a result of shear deformations in the course of drying down from suspension in solvent [1]. For it is clear that stress will be concentrated at boundaries between the deformed, and as yet undeformed, regions of these crystals and may generate the dislocations required for this process. In later stages of collapse of an initially pyramidal crystal, continuing collapse could be brought about by a screw dislocation of type 1 circuiting the boundary of the undeformed region in an ever decreasing spiral.

Repeated passage of dislocations along the same (010) glide plane in a case such as shown in figure 4, however, would have the effect of partly disrupting the structure but leaving those parts of the crystal which lie on opposite sides of the glide plane connected by sections of alined chains. If such a process were to occur within a chain folded crystallite in bulk polyethylene it would doubtlessly be accompanied by a reorientation of the molecular chain about the $a$ axis as is often observed in fibers drawn to moderate elongations [12].

It is convenient at this point to consider briefly the questions of the climb motion and the intersection of dislocations $[8,9]$. Our remarks are applicable not only to polymer crystals but also to the infinite chain crystal and to hydrocarbon crystals of appreciable chain length $(n>20$, say). First we note that climb involves diffusion of vacancies or interstitials, but in the conventional sense these defects have no significance in the various crystals under consideration. (Vacancies could exist at chain ends in a polymer crystal, but would be immobile in the sense that they cannot diffuse independently of the specific chains with which they are associated.) Since diffusional motion in these structures would require the displacement of entire chains (or of extended lengths of chain in a polymer crystal) dislocation climb will be an insignificant process except perhaps in chain-folded crystals at elevated temperatures.

The intersection of dislocations in long chain molecular crystals would require considerable space for thorough discussion. Suffice it to say that we have examined all possible intersections of the dislocations listed in table 1 , and that the results may be summarized as follows. Intersections lead in general to the formation of jogs, which in some instances offer little resistance to the further motion of dislocations and in other instances immobilize dislocations by being constrained to move only by climb. The climb of such a jog is forbidden for the reason that it would involve the diffusion, not of entire chains or extended lengths of chain, but of interstitials or vacant lattice sites. Certain inter-

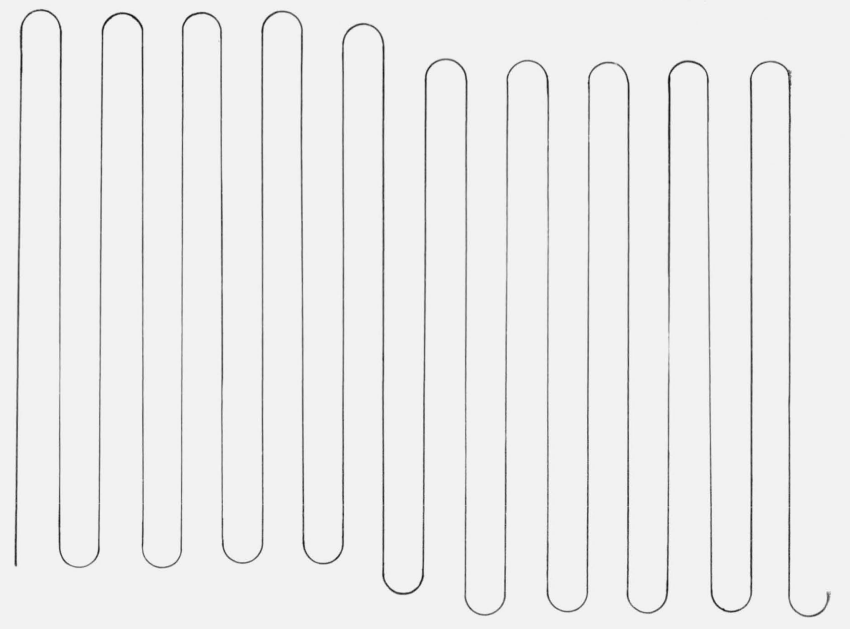

FrguRE 4. A schematic representation of the configuration of a chain folded molecule in a polyethylene crystal after the passage of a dislocation with [001] Burgers vector.

sections are physically impossible since, as in the case of dislocation 3 intersecting dislocation 5 , they would lead to jogs which cannot be formed without primary bonds being broken. In this situation the dislocations are pinned. The following rules apply. Half of the conceivable screw-screw intersections are physically impossible and only those are allowed in which a dislocation of type 1 intersects dislocations of types 2, 3, and 4. Even in these cases, the first dislocation acquires a jog which has to glide out of the crystal along the screw axis before the dislocation as a whole can glide; the other dislocation $(2,3$, or 4$)$ is totally immobilized. Edge dislocations, on the other hand, may intersect one another without immobilization. Of the 17 conceivable screw-edge intersections, 7 are physically impossible, 7 involve immobilization of the screw, and in only 3 can the screw and edge both move freely after intersection. In hydrocarbon crystals, of course, some of these restrictions are relaxed on account of the presence of unique glide planes lying between molecular layers.

To summarize, it is clear that dislocations are unlikely to play a major part in bringing about deformations of appreciable magnitude in chainfolded polymer crystals. As we have seen, repeated slip on a given glide plane is impossible unless there are attendant readjustments in the disposition and packing of folds. Our remarks concerning intersections make it evident that extended movement of dislocations will in general be severely restricted. Moreover, the relatively small number of dislocations likely to be present initially in polymer crystals as grown (whose volumes are usually small, $10^{-12} \mathrm{~cm}^{3}$ or less) would soon be used up in quite small deformations; and we note that their replacement by mecha- 
nisms such as might operate in metal crystals is in the present case highly improbable. A Frank-Read source [13], for example, requires for its operation an area of glide plane of the order of a $1 \mu$ square, and the only planes of sufficient extent in chain-folded polymer crystals are not permissible glide planes.

A possible deformation mechanism which could give rise to substantial and more or less uniform shear strains in these crystals is twinning. This involves a rearrangement in packing requiring no more than a slight translation of one chain relative to its neighbors, and what little evidence is currently available does indeed suggest that deformation by twinning is a common occurrence [2, 14]. As has already been pointed out, in chain-folded polyethylene crystals the only unrestricted glide planes are the fold planes. Thus in these crystals twinning would be expected to occur on (110) planes, and this does appear to be the case [14]. On the other hand, in oriented bulk polyethylene, where regular chain folding does not exist, other twin planes are possible, and (310) twins have been reported [2]. Here, as in simpler solids, the mechanism by which twinning occurs remains uncertain; the successive generation and motion of dislocations on adjacent glide planes may be involved, but, of necessity, it must be a cooperative process.

Very large plastic deformations, such as occur in the drawing of fibers, most likely involve various complex catastrophic processes. But, in the event that tensile stress is applied normal to the $c$ axis of a crystal, one might expect a simple "unzippering" of folded chains to give rise to fine fibrillar texture, a process for which there is already some experimental evidence $[15,16]$.

It seems reasonable to conclude that appreciable deformations of chain-folded polymer crystals cannot be attributed solely or simply to dislocation mechanisms. Molecular rearrangements other than slip in an otherwise undisturbed lattice must be involved, and these require careful consideration. Dislocations may make important contributions to plastic deformation in hydrocarbon crystals, however, or in "extended chain" polymer crystals such as have recently been reported in some samples of bulk polyethylene [17].

We are very grateful to Drs. F. Khoury of the National Bureau of Standards and P. H. Lindenmeyer of the Chemstrand Research Center for many critical comments and numerous helpful discussions.

\section{References}

[1] H. D. Keith "Crystallization of long-chain polymers" in The Physics and Chemistry of the Organic Solid State, ch. 8, Interscience, New York (1963).

[2] F. C. Frank, A. Keller, and A. O'Connor, Phil. Mag. 3, 64 (1958)

[3] D. A. Zaukelies, J. Appl. Phys. 33, 2797 (1962); M. F. Bender and M. L. Williams, J. Appl. Phys. 34, 3329 (1963).

[4] D. H. Reneker, J. Polymer Sci. 59, S39 (1962).

[5] C. W. Bunn and T. C. Alcock, Trans. Faraday Soc. 41, 317 (1945).

[6] A. Keller, Phil. Mag. 6, 329 (1961).

[7] D. C. Bassett and A. Keller, Phil. Mag. 6, 345 (1961).

[8] W. T. Read, Dislocations in crystals (McGraw-Hill Book Co., Inc., New York, N.Y., 1953).

[9] A. M. Cottrell, Dislocations and plastic flow in crystals (Oxford, 1953)

[10] P. W. Teare, Acta. Cryst. 12, 294 (1959).

[11] D. H. Reneker and P. H. Geil, J. Appl. Phys. 31, 1916 (1960).

[12] S. L. Aggarwal and O. J. Sweeting, Chem. Rev. 57, 665 (1957).

[13] F. C. Frank and W. T. Read, Phys. Rev. 79, 722 (1950).

[14] P. H. Geil (in press).

[15] A. Keller, Phil. Mag. 2, 1171 (1957).

[16] H. D. Keith and F. J. Padden, Jr., J. Polymer Sci. 41, 525 (1959)

[17] P. H. Geil, F. R. Anderson, B. Wunderlich, and T. Arakawa (in press).

(Paper 68A5-297) 